ÉTUDES

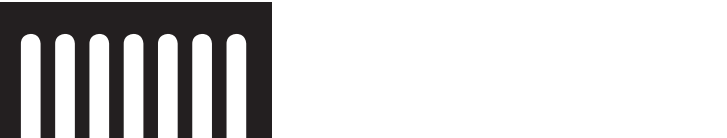





\title{
La multiplicité linguistique au service de l'expression de l'unité : les différentes traductions du mot un en tchèque
}

\section{Linguistic Multiplicity Serving Unit Expression: Different Czech Translations of the Word un}

\author{
KRISTínA KolÁčKovÁ [342701@mail.muni.cz] \\ Masarykova univerzita, République tchèque
}

\section{RÉSUMÉ}

Sans distinction préalable entre un article indéfini et un adjectif numéral cardinal, défendue par l'écrasante majorité des grammaires, nous cherchons à analyser différents moyens d'expression de ce déterminant en tchèque, langue qui ne possède pas de système des articles en tant que tel ; pour ce faire, nous recourons à un échantillon tiré du corpus plurilingue InterCorp. Par l'intermédiaire des traductions, nous tentons également à démontrer que la prétendue dichotomie de ce déterminant français n’est pas toujours si évidente : dans ce but, nous nous servons entre autres d'une théorie du système des déterminants de Marc Wilmet, basée sur le concept d'extensivité. (102 mots)

\section{MoTS CLÉS}

Un article indéfini ; un adjectif numéral cardinal ; théorie de Marc Wilmet ; extensivité ; expression de un en tchèque; distinction entre un article et un numéral

\begin{abstract}
Disregarding the established distinction between the indefinite article un and the cardinal numeral un, supported by the overwhelming majority of grammars, the paper attempts to analyse the different ways of expressing this determinant in Czech language, which does not have the system of articles as such. To do this a sample extracted from the parallel corpus InterCorp is used. By means of the translations the paper also tries to demonstrate that the alleged dichotomy of this French determinant is not always that unequivocal; here the author makes use of Marc Wilmet's determinant system theory, among others, which is based on the concept of extensivity. (104 words)
\end{abstract}

\section{KEYWORDS}

Indefinite article un; cardinal numeral un; Marc Wilmet's theory; "extensivité" (extensivity); expression of $u n$ in Czech; distinction between article $u n$ and numeral un

REÇU 2015-6-15 ; ACCEPTÉ 2016-02-01 


\section{Introduction}

Risquons un truisme pour initier notre raisonnement : "Un n'est pas toujours un. » Du moins d'après ce qui est généralement admis - il suffit de jeter un coup d’oeil dans l'écrasante majorité des grammaires - il s'agit de deux différents types de déterminants, le plus souvent appelés un article indéfini et $u n$ adjectif numéral cardinal ${ }^{1}$.

Pour autant, la distinction entre eux ne semble pas être claire et nette (nous essayerons de le démontrer dans la partie «Un article vs. un numéral »). C’est pourquoi, lors de la réalisation de l'objectif de cet article ${ }^{2}$, une analyse de différents moyens d'expression de ce petit mot en tchèque, nous ne tiendrons presque pas compte de cette dichotomie, mais recourrons plutôt à une théorie du système des déterminants innovante, à savoir celle du linguiste belge Marc Wilmet que nous présentons brièvement ci-dessous.

\section{Théorie wilmetienne : un et l'extensivité}

Marc Wilmet distingue lui aussi deux types de un : le premier, équivalent de un article, relève d'après son classement du groupe des quantifiants bipolaires ${ }^{3}$, le second, équivalent de un numéral, du groupe des quantifiants numéraux. Néanmoins, la différence n’est pas seulement d'ordre terminologique : elle repose essentiellement sur l'introduction du concept d'extensivité, qui joue un rôle crucial dans le système wilmetien des quantifiants.

Toutefois, avant de traiter de un quantifiant bipolaire, il sera sans doute utile d'expliquer les termes, tout d'abord celui de l'extensivité qui exprime le rapport entre :

- l'extensité = quantité d'êtres ou d'objets auxquels un nom (ou un groupe nominal) est appliqué, et

- l'extension (en énoncé) = ensemble des êtres ou des objets auxquels le nom (ou le groupe nominal) est contextuellement applicable (Wilmet 2007 : 133).

Deux cas de figures peuvent donc survenir (ibid.) :

- soit l'extensité épuise l'extension : dans ce cas il s'agit de l'extensivité extensive,

- soit l'extensité est inférieure à l'extension : on a alors affaire à l'extensivité partitive.

Pour une meilleure compréhension de la théorie, illustrons à présent ce qui vient d'être dit (les exemples proviennent de l'ouvrage de l'auteur (2007: 134)) :

1 En effet, dans le présent article, nous traitons du mot un exclusivement en tant que déterminant (c'est-à-dire en tant qu'article ou adjectif numéral cardinal) et écartons un employé comme adjectif numéral ordinal/nom masculin invariable/adjectif qualificatif/pronom (distinction tirée de l'entrée «Un, une » du dictionnaire Le Petit Robert). Bien évidemment, toutes nos remarques à propos de ce déterminant sont valables tant pour la forme masculine un que pour la forme féminine une.

2 Pour élaborer cet article, nous avons puisé essentiellement de notre mémoire de master Le mot UN en questions (2014).

3 Pour l'integralité de la présentation des quantifiants bipolaires, consulter : Wilmet (2007: 125-176). 
1. Un homme entra, qui avait l'air hagard.

2. L'homme était entré et s'était assis au coin du feu.

Dans la première phrase, « homme » en question est extrait d’un ensemble pluriélémentaire des humains du sexe masculin (extensité $=1$, extension $=n^{4}>1$ ). Dans la deuxième phrase, l'extension de « homme » étant préalablement modifiée - autrement dit réduite à un ensemble singleton, en l'occurrence à un individu - on reprend cet élément unique et on l'installe dans l'univers du discours (extensité $=1$, extension $=1$ ). Ainsi, dans la première phrase, nous avons affaire à l'extensivité partitive (extensivité $=1 / n$ ), alors que « homme » dans la deuxième phrase marque une extensivité extensive (extensivité $=1 / 1$ ).

Regardons un autre couple de phrases (de même repris de l'auteur (2007 : 134)), exprimant à leur tour une vérité générale :

\section{L'homme est mortel.}

4. Un enfant est toujours l'ouvrage de sa mère.

Dans la troisième phrase, l'extensité de « homme » (extensité $=n>1=t$ ) est égale à son extension collective maximale (extension $=t$ ), on obtient donc - conformément à la théorie l'extensivité extensive (extensivité $=t / t)$. Mais que faire de la quatrième phrase qui n'entre apparemment pas dans l'idée de l'extensivité partitive ? Pour éclairer ce point, Wilmet (2007 : 149) soutient que un, n'ayant pas en lui-même la capacité de dépasser l'extensité égale à 1, peut la tirer soit de la prédication universelle ${ }^{5}$ (pour rendre compte de cette éventualité, le linguiste se sert du mécanisme $1 \times n \rightarrow t$ ) - ce qui est le cas de la phrase $\mathrm{n}^{\circ} 4$, où « la prédication récurrente est louvrage de sa mère multiplie l'extensité individuelle de enfant autant de fois que nécessaire pour accéder à l'extensité collective maximale » (2007: 134) -, soit d'un déclencheur cotextuel (pour ce cas, Wilmet pose le mécanisme $1+1+1 \ldots+1=n<t)^{6}(2007: 149)$, ce que nous pouvons illustrer par les exemples suivants (2007 : 151-152) :

5. La plupart des filles aiment un garçon.? (Rem. : Ici, le déclencheur de l'extensité croissante est un quantifiant donneur.)

6. Chaque jour, à chaque heure, à chaque minute, à chaque seconde, une voiture écrase un piéton. (Rem. : Dans cette phrase, le déclencheur prend la forme d'une complémentation itérative.)

4 Le signe « $n$ » signifie le nombre d’unités et le signe « $t$ » signifie la totalité.

5 Wilmet élucide le terme de prédication universelle, qui est en opposition avec la prédication existentielle, comme suit (2007 : 149) : «La prédication universelle - [...] - étend la validité de l'énoncé à tous les mondes possibles (énoncés dits " analytiques", dont la vérité est indépendante du locuteur : Deux et deux font quatre, etc.) et à tous les univers de croyance (énoncés dont la vérité dépend du locuteur : Les chats sont intelligents, etc.) : p. ex. Un chien aboie = «il existe un $x$ tel que si $x$ est chien, alors $x$ aboie » [...]."

6 En parlant des déclencheurs cotextuels, il convient de préciser que l'extensité dans ces cas n'est pas forcément maximale, mais qu'elle est en tout cas supérieure à 1 .

7 Certes, plusieurs interprétations sont possibles, parmi lesquelles celle qu'on pourrait paraphraser en « chaque fille ou presque a son amoureux» $(2007: 151)$. 
7. Dupont chasse une perdrix de grande taille. (Rem. : Ici, c'est la caractérisation du nom receveur qui permet d'augmenter l'extensité de « perdrix».)

La valeur d'origine du quantifiant bipolaire $u n$ - héritée de son prédécesseur latin $u n u s^{8}-$ relève donc de l'extensité égale à 1, c'est-à-dire de l'extensivité partitive. Suivant le contexte, l'extensité de un peut dépasser une unité et ainsi augmenter l'extensivité de ce quantifiant (voir le tableau ci-dessous).

\begin{tabular}{|c|c|c|}
\hline $\mathbf{N}^{\circ}$ & Valeur de l'extensité & Type de l'extensivité \\
\hline 1 & $\mathrm{t}>$ extensité $=1$ & \multirow{2}{*}{ extensivité partitive } \\
\hline 2 & $\mathrm{t}>$ extensité $>1$ & \\
\hline 3 & $\mathrm{t}=$ extensité $>1$ & extensivité extensive \\
\hline
\end{tabular}

Fig. 1 : Extensité vs. extensivité

En ce qui concerne un quantifiant numéral, les choses paraissent moins compliquées : conformément à la caractéristique du groupe auquel il appartient, il « déclar[e] une extensité chiffrable » (2007 : 187), c'est-à-dire l'extensité égale à 1 .

Nous allons à présent mesurer la pertinence de ces outils théoriques au cours d'un essai d'analyse contrastive français-tchèque.

\section{Différentes possibilités de l'expression de un en tchèque}

Deux questions principales régissent notre recherche empirique :

- Par quels moyens le déterminant un (sans distinction préalable entre un article et un numéral) est-il exprimé en tchèque?

- Que peut-on induire - par l'intermédiaire des traductions - de la distinction entre un article et un numéral ?

Dans ce but, nous avons décidé de nous servir de la version 6 du corpus InterCorp ${ }^{9}$, que l’on peut définir comme un corpus plurilingue parallèle non-référentiel ${ }^{10}$, élaboré depuis 2005 dans le cadre du projet Český národní korpus (ČNK $)^{11}$. Il englobe des textes en langue tchèque

8 Pour en savoir plus sur l'évolution de l'article un, consulter Carlier (2001).

9 Pour plus d'informations sur ce corpus, voir : « Korpus InterCorp », disponible à l'adresse : http://ucnk.ff.cuni.cz/ intercorp/?req=page:info (consulté le 2 avril 2014).

10 C'est-à-dire que l'ensemble de textes qui le constituent augmente continuellement.

11 «Paralelní korpus InterCorp», disponible à l’adresse : http://wiki.korpus.cz/doku.php/pojmy:paralelni (consulté le 2 avril 2014). 
et leurs traductions dans d'autres langues (une trentaine de langues au total) ${ }^{12}$. En général, les textes, de différents genres, sont récents (les plus anciens datent de 1945) et sont tous alignés par paragraphe, voire par phrase ${ }^{13}$. C’est pourquoi il s'agit sans doute d'un moyen utile pour mener une enquête contrastive telle que la nôtre.

Étant donné les possibilités et les écueils ${ }^{14}$ que constitue l'utilisation de ce corpus, notre recherche ne peut ni ne prétend relever d'aucune ambition statistique, mais veut plutôt illustrer les propos de son auteur. Obligée d'examiner au cas par cas l'échantillon choisi, nous n'avons pas pu exploiter les données du corpus d'une façon exhaustive.

\subsection{Le choix de l'échantillon}

Nous avons opté pour le noyau du corpus qui est constitué principalement de textes littéraires alignés « à la main ", à la différence de la collection, composée de textes journalistiques, de droit et autres, alignés automatiquement ${ }^{15}$. En ce qui concerne la langue source des textes, nous avons choisi le français au regard de nos objectifs.

En effet, nous voulons plutôt étayer nos constats et nos hypothèses : notre échantillon principal (appelé dès lors Échantillon 1), formé par une sélection aléatoire du noyau de la partie française d'InterCorp (exclusivement de textes en langue source française) ${ }^{16}$, contient au total 500 occurrences de un (ne faisant pas distinction entre un article et un numéral). Voici ce que, à notre sens, on peut induire de son examen.

\subsection{Expression de un en tchèque}

Comme l'affirme le linguiste tchèque Otomar Radina, qui s'est - à notre connaissance - peutêtre le plus intéressé à la traduction de un, dans son ouvrage Francouzština a čeština: systémové srovnání dvou jazykủ (1977), la langue tchèque, ne possédant pas de système des articles sous une forme distincte, est amenée à exprimer les valeurs des articles français dans trois domaines : lexical, morphologique et syntaxique, l'un se combinant souvent à l'autre (1977: 18). Nous allons essayer d'illustrer ce constat en l'appliquant auxdits un article et un numéral.

\footnotetext{
12 Ibid.

13 Ibid.

14 L’un d'eux est la taille de la partie française du corpus qui, dans son noyau, comporte - en comparaison avec d'autres langues telles que l'anglais, l'allemand ou l'espagnol - « seulement » 3816000 mots (" Korpus InterCorp ", disponible à l’adresse : http://ucnk.ff.cuni.cz/intercorp/?req=page:info (consulté le 2 avril 2014)).

15 Ibid.

16 Les textes en langue source française de la partie française d'InterCorp contiennent 32019 occurrences de un.
} 


\subsubsection{Niveau lexical}

Après avoir éliminé les occurrences de un qui ne correspondaient ni à un numéral ni à un article (c'est-à-dire les occurrences de un employé comme pronom ou comme adjectif qualificatif, les occurrences du numéral « vingt et un » et les concordances ${ }^{17}$ mal alignées), l'Échantillon 1 s'est réduit à 466 occurrences. Nous avons relevé 64 occurrences (donc presque $14 \%$ de 466 occurrences) où un a été traduit " directement ${ }^{18}$ au niveau lexical : nous les avons réparties en 11 types d'expressions différentes (rangées d’une façon descendante par nombre d'occurrences) : jeden, nějaký, jakýsi, celý, jediný, takový, nový, úplný, každý, určitý, jednou (O. Radina mentionne par contre - au niveau lexical - 10 expressions suivantes : jeden, takový, nějaký, jakýsi, kterýsi, někde, někdy, úplný, hotový, učiněný) (1977 : 32)). À titre d’exemple, nous avons choisi quatre cas particulièrement intéressants :

- jeden

«Un jour, je tombai sur une photo que je n'aurais pas dû voir...» (Andreï Makine, Le Testament français)

« Jednoho dne jsem narazil na fotografii, kterou jsem vidět neměl... (Andreï Makine, Francouzský testament, traduit par Vlasta Dufková) ${ }^{19}$

L'expression lexicale « directe » incontestablement la plus fréquente (presque dans la moitié d’occurrences relevées) était celle à l'aide du numéral jeden.

- nový

«C'est que l'instituteur, ayant pris sa retraite, avait été remplacé par une institutrice étrangère au pays, et tout le monde l'observait pour savoir de quel bois elle était faite. » (Michel Tournier, Le coq de bruyère)

« Nebot na místo učitele, který šel do penze, nastoupila nová učitelka, původem odjinud, a všichni ji sledovali a zjištovali, z jakého je těsta. » (Michel Tournier, Tetřev hlušec, traduit par Václav Jamek)

Du point de vue référentiel, la traduction à l'aide de nový est en accord avec une des caractéristiques qu'on donne de l'article dit indéfini où un sert à introduire un nouveau référent dans le monde du discours (Carlier $2001: 69$ ).

17 En linguistique de corpus, une concordance est en fait une occurrence du mot recherché avec son contexte droit et gauche (formant le plus souvent une phrase ou un paragraphe) («Konkordance », disponible à l'adresse : http://wiki. korpus.cz/doku.php/pojmy:konkordance (consulté le 3 avril 2014)).

18 Directement entre guillemets, car, pour pouvoir traduire un énoncé, il est nécessaire de se rendre compte d'une synergie du plan lexical, morphologique et syntaxique, les expressions dépendant les unes des autres et co-construisant toutes ensemble le sens de l'énoncé.

19 Dans la suite de l'article, lorsque nous citons des exemples de langue source française tirés d'InterCorp, les expressions tchèques servant de traduction à un (ou d'expression de ses valeurs) sont mises en gras ou en italique par nous-même. 


\section{- úplný}

« Car pour cet homme tout en dehors, le repli ne pouvait être qu'une démission et la vieillesse un naufrage. » (Michel Tournier, Le coq de bruyère)

«Pro tohoto muže žijícího jen pro vnější svět totiž každý ústup z pozic vyzníval jako kapitulace, a stáŕí jako úplný krach. » (Michel Tournier, Tetřev hlušec, traduit par Václav Jamek)

Úplný au sens figuré (remarquons qu'au sens premier il marquerait plutôt une extensivité extensive), qu'on aurait traduit plutôt p. ex. par « un véritable », fait aussi partie de la liste d'expressions servant de traduction de un proposée par O. Radina : c'est pourquoi nous le conservons également dans notre « inventaire».

\section{- $k a \check{z} d y$}

«Il répond qu'il y va tout de suite, qu'il joue pour faire plaisir aux autres, mais qu’il déteste qu'on ergote sur un point, qu'on l'asticote pour avoir jeté trop tard son roi de carreau et qu'on lui vole ses sous comme à un pigeon. » (Sébastien Japrisot, Un long dimanche de fiançailles)

« Odpoví jí, že tam půjde hned, stejně hraje jen proto, aby udělal radost ostatním, nesnáší, když se někdo hašteří pro každou maličkost, sekýruje ho, že hrál kárového krále moc pozdě, a obírá ho o peníze jak hejla.» (Sébastien Japrisot, Př́liš dlouhé zásnuby, traduit par Veronika Sysalová)

Il est à noter - du point de vue de la théorie wilmetienne - que dans l'exemple en question, každý reflète fidèlement l'extensité maximale de un point due à la prédication universelle.

Certes, loin de déclarer nos résultats représentatifs vu la taille de notre échantillon, nous les citons à titre d'illustration des différentes possibilités d'expression lexicale de un en tchèque : peut-être en existe-t-il d'autres car elles dépendent considérablement du contexte. En effet, il est intéressant de voir comment le sens global de lénoncé - co-construit par toutes les expressions composantes de l'énoncé et par le contexte de cet énoncé ${ }^{20}$ - favorise telle ou telle expression en tchèque pour traduire un français : par exemple au cas de nový (expression $\mathrm{n}^{\circ} 7$ ci-dessus), le verbe remplacer convoque - généralement - un élément nouveau, ce qui se manifeste en tchèque par la traduction de une institutrice par nová učitelka. Néanmoins, dans un autre contexte, la traduction de un à l'aide de nový pourrait - bien sûr - ne pas être juste.

Ceci nous permet de renouer avec les cas où l'expression de la valeur de un, bien qu'au seul niveau lexical, se fait d'une façon clairement «indirecte ", souvent ensemble avec l'appui de la traduction d'une ou plusieurs autres expressions composantes de l'énoncé et souvent au moyen d'une autre partie du discours qu'est l'adjectif (du point de vue de la grammaire française). En voici quelques exemples (pris de l'Échantillon 1) :

20 Pour se renseigner davantage sur une théorie fort intéressante de la construction de la signification d'un énoncé, voir l'article de Franckel (1998: 61-84). 
"Un détail imperceptible et la fusion opère, ou pas. »(Pierre Assouline, Double vie)

"Stačí nepostřehnutelný detail, a dojde ke splynutí, nebo ne. "(Pierre Assouline, Dvojí život, traduit par Šárka Belisová)

Le traducteur a opté pour le verbe stačit (qu’on pourrait éventuellement sous-entendre aussi dans la phrase française : il suffit), qui est bien compatible avec l'idée de l'extensivité partitive.

« Ainsi, lorsque l'amour s'introduisait dans mes pensées, c'était sous forme de tentation et je ne pouvais l'envisager autrement que comme une sorte de déchéance. » (Michel Leiris, L'âge d'homme)

«A tak když se láska vetřela do mých myšlenek, bylo to jako pokušení a já na ni nemohl hledět jinak než jako na jistý úpadek. » (Michel Leiris, Věk dospělosti, traduit par Kateřina Vinšová)

Une sorte de, de même que une espèce de, un genre de, une manière de, etc. - dans la terminologie de Wilmet appelés des quantifiants-caractérisants stricts, agissant à la fois sur l'extension et l'extensité du nom - servent à corriger une identification abusive (Wilmet 2007 :239), ainsi que le fait l'adjectif jistý en tchèque.

« Mais après un silence il me lança, avec une sorte de rancune [...] » (Antoine de Saint-Exupéry, Le petit prince)

"Ale po chvilce mlčení odsekl trochu nevraživě [...] » (Antoine de Saint-Exupéry, Malý princ, traduit par Zdeňka Stavinohová)

Silence, a priori perçu comme innombrable, est employé avec un article indéfini qui est - dans cette phrase - à l'origine de sa restitution «numérative » : d'où l'emploi du mot chvilka en tchèque, exprimant un intervalle de temps voué au silence.

\subsubsection{Niveau morphologique}

Même si O. Radina ne parle pas de ce niveau en ce qui concerne l'expression de un, il existe pourtant bien. Soit l'expression morphologique de un se combine avec son expression lexicale (ce que nous illustrerons plus loin), soit un n'est exprimé que sur le plan de la morphologie, comme c'est le cas dans les exemples suivants (de nouveau extraits de l'Échantillon 1):

«Celle qui vous trouble au zoo devant les survivants d'une espèce éteinte. » (Antoine de SaintExupéry, Lettre à un otage)

«Takovou, která se vás zmocňuje v zoologické zahradě před posledními představiteli vymírajících druhů. » (Antoine de Saint-Exupéry, Dopis rukojmímu, traduit par Zdeňka Stavinohová)

Le changement du nombre grammatical en tchèque correspond à la valeur générique de un en français (dans les mots de Wilmet, le syntagme une espèce éteinte dépasse l'extensité égale à 1). 
« Aux journalistes sélectionnés, Gravelin répétait cependant combien le vieillard avait un bon fonds $[$ sic] : ne donnait-il pas, chaque année, la moitié de ses revenus à un organisme de charité ?» (Amélie Nothomb, Hygiène de l'assassin)

« Přesto Gravelin vybraným novinářum opakovaně tvrdil, jaký je stařec z gruntu dobrák: cožpak nevěnuje každoročně polovinu svých honorářů charitativním organizacím? » (Amélie Nothomb, Vrahova hygiena, traduit par Jarmila Fialová)

Le même phénomène a lieu dans la flexion du nom.

«Chaque pas qu'il faisait arrachait à Balthasar un profond soupir car chaque pas l'éloignait de sa chère Ursula, et pour combien de temps? " (Frédérick Tristan, Les tribulations héroïques de Balthasar Kober)

«Baltazar si při každém kroku hluboce povzdychl, protože se vzdaloval od milované Uršuly a kdo ví, na jak dlouho...» (Frédérick Tristan, Hrdinné útrapy Baltazara Kobera, traduit par Oldřich Kalfiřt)

Cette fois-ci, l'extensivité partitive de un se traduit en tchèque - qui est une langue hautement flexionnelle - dans la flexion du verbe, précisément par l'emploi du verbe povzdychnout si, formé du préfixe po- et du suffixe -nout, les deux indiquant l'aspect perfectif.

Un autre exemple (tiré d'InterCorp) illustrant l'expression de un dans le domaine de la flexion verbale (la valeur générique de un y est rendue par l'emploi d'une forme verbale à sens itératif bývá) :

«À l'entrée d'une grotte, il y a la lumière. » (Pierre Assouline, Double vie)

«U vchodu do jeskyně bývá světlo. » (Pierre Assouline, Dvojí život, traduit par Šárka Belisová)

\subsubsection{Niveau syntaxique}

Du point de vue de la perspective fonctionnelle de la phrase, l'ordre des mots peut aider à identifier le thème et le rhème ${ }^{21}$ : en tchèque, on observe une tendance à placer les éléments les plus rhématiques (et les syntagmes nominaux avec un le sont souvent) vers la fin de l'énoncé22 (exemples tirés de l'Échantillon 1):

21 Thème et rhème, deux notions qui vont de paire et qui sont cruciales pour la perspective fonctionnelle de la phrase. Vilém Mathesius, fondateur de la théorie de la perspective fonctionnelle de la phrase, en donne la définition suivante : «A closer examination of sentences from the viewpoint of assertiveness shows an overwhelming majority of all sentences to contain two basic content elements: a statement and an element about which the statement is made. [...] The element about which something is stated may be said to be the basis of the utterance or the theme, and what is stated about the basis is the nucleus of the utterance or the rheme. " (Mathesius 1975: 81).

22 En français, c'est aussi le cas, néanmoins des contraintes syntaxiques peuvent s'imposer : par exemple lorsqu'un sujet est un élément rhématique, dans un énoncé non marqué il est placé au début de celui-ci (comme c’est le cas dans le deuxième exemple cité : « [...] une joie engouffra son visage $[\ldots] »)$. 
«Il n’y a pas une grande différence entre consommer et consumer. » (Frédéric Beigbeder, 99 francs)

« Mezi slovy konzumovat a stravovat není velký rozdíl. » (Frédéric Beigbeder, 99 franki̊, traduit par Markéta Demlová)

«Cette dernière levait déjà sur lui un regard de western, quand, inattendûment, paupières en surprise, une joie engouffra son visage [...] » (Patrick Chamoiseau, Solibo Magnifique)

«Ta už se chystala zdrtit ho kovbojkovým pohledem, když tu najednou překvapením zvedla víčka a tvář jí zaplavila radost [...] » (Patrick Chamoiseau, Solibo Ohromný, traduit par Růžena Ostrá)

«Vous me le disiez vous-même tout à l'heure, c'est une cause mystérieuse qui est venue bloquer votre plume. » (Amélie Nothomb, Hygiène de l'assassin)

«Sám jste mi před chvílí řekl, že vaše pero se zablokovalo ze zcela tajemného důvodu. » (Amélie Nothomb, Vrahova hygiena, traduit par Jarmila Fialová)

Lordre des mots y étant beaucoup plus flexible qu'en français, le tchèque n’est pas obligé d'avoir recours à la focalisation (rhématisation) comme dans la phrase d’origine.

\subsubsection{Combinaisons des niveaux d'expressions}

\section{Combinaison de l'expression lexicale avec l'expression morphologique}

L'expression lexicale de un se combine souvent avec l'expression au niveau morphologique (exemple tiré de l'Échantillon 1) :

«Il y avait un claquement sec : mon arrière-petit-neveu, là-haut, fermait son livre : il rêvait à l'enfance de son arrière-grand-oncle et des larmes roulaient sur ses joues [...]» (Jean-Paul Sartre, Les Mots)

« Něco suše zapraštělo: někde nahoře zavřel můj praprasynovec knihu: zasnil se nad dětstvím svého prastrýce a po tvárích mu stékaly slzy [...] » (Jean-Paul Sartre, Slova, traduit par Dagmar Steinová)

Le pronom něco, conforme à l'extensivité partitive de un, produit avec le verbe zapraštět à sens perfectif (créé grâce au préfixe $z a-$ ) un effet de sens semblable que celui de la phrase de départ.

\section{Combinaison de l'expression morphologique avec l'expression syntaxique}

«Lee, emmenez-moi faire un tour avant le déjeuner. » (Boris Vian, J'irai cracher sur vos tombes)

«Lee, pojdte se se mnou před obědem projet. » (Boris Vian, Naplivu na vaše hroby, traduit par Martin Řízek et Petr Turek) 
Faire un tour, traduit comme projet se, a un sens assez général (dans les mots de Wilmet, il relève de l'extensivité partitive) à la différence de faire le tour, d'habitude accompagné d'un complément : faire le tour du monde, de Paris, de la maison, etc. (InterCorp). En plus, dans la phrase tchèque, il se trouve à la fin de l'énoncé, car il s'agit bien de l'élément le plus rhématique.

\section{Combinaison de l'expression syntaxique avec l'expression lexicale}

«Il n'y avait qu'un mot pour résumer mon état : « confusion ».» (Pierre Assouline, La cliente)

«Stav, v němž jsem se nacházel, se dal popsat pouze jediným slovem: zmatek. » (Pierre Assouline, Zákaznice, traduit par Lubomír Martínek)

Jediný, sans doute conditionné par la présence de ne...que dans la phrase de départ, se situe avec le nom auquel il se rapporte - vers la fin de l'énoncé, le complément de nom mot exprimé en tchèque au début de l'énoncé.

\section{Combinaison des trois niveaux d'expression}

Même si nous n'avons pas trouvé d'exemple dans l'Échantillon 1 qui le prouverait, on ne peut pas exclure cette éventualité.

\subsubsection{Aucune expression explicite}

Très souvent (presque dans $80 \%$ des occurrences de notre Échantillon 1), on ne trouve aucune forme d'expression explicite de $u n$. Il en est ainsi par exemple dans l'extrait suivant :

«Si j’ai le temps de te laisser un autre message après ma consultation, je le ferai... Je dois raccrocher parce qu'un agent, au feu rouge, n'arrête pas de me fixer» (Pierre Assouline, Double vie)

« Jestli mi to vyjde, ještě ti nechám zprávu, až budu mít po ordinaci... Musím končit, protože [Ø] strážník na křižovatce po mně pořád pokukuje. » (Pierre Assouline, Dvojí život, traduit par Šárka Belisová)

Somme toute, à moins de ne pas être visiblement exprimé dans l'énoncé tchèque, ce déterminant trouve son expression sur un ou plusieurs plans (lexical, morphologique, syntaxique), le moyen concret dépendant avant tout des choix du traducteur.

Maintenant, nous pouvons prêter l'attention à notre analyse de l'Échantillon 1 ciblée sur la question de distinction entre deux types de $u n$. 


\subsection{Un article vs. un numéral}

"Cette question est en débat depuis les premières grammaires du français. Il s'agit de savoir si un/une est toujours numéral, ou si deux types d'emploi doivent être distingués : numéral vs article » (Fournier 2005 : 113). D’après ce que nous avons pu constater lors de l'étude de la problématique de un, cette distinction est maintenue dans la plupart des ouvrages linguistiques et grammaticaux, même si elle reste peu traitée. Dans nos analyses, jusqu'ici, nous n'en avons presque pas tenu compte. Il en était ainsi parce qu'apparemment, il n'existe pas - ou bien, à notre connaissance, il n'a pas encore été découvert - un critère objectif et universel pour distinguer les deux (le critère sémantique n'étant pas assez fiable vu sa relative subjectivité). Il semble que seule la présence de certaines expressions, telles que ne... $q u e^{23}$, pas (un), seul, etc. ${ }^{24}$, puisse indiquer clairement qu'il s'agit de un quantifiant numéral (dans la terminologie de Wilmet). Sinon, un article peut faire ressortir la valeur numérale avec plus ou moins de force : néanmoins, de la part des locuteurs, la perception de cette valeur peut varier, dont témoignent d'ailleurs certains exemples de traduction (tirés de l'Échantillon 1):

« Nous imaginions un vénérable vieillard - unissant dans ses traits la noble prestance de notre arrière-grand-père Norbert et la solennité pharaonique d'un Staline -, un vieillard à la barbe chenue, assis devant une table tristement éclairée par une bougie. » (Andreï Makine, Le Testament français)

«Představovali jsme si ctihodného kmeta - jeho rysy v sobě spojovaly ušlechtilou impozantnost pradědečka Norberta a faraonskou okázalost takového Stalina -, kmeta se sněhobílým vousem, jak sedí u stolu žalostně osvětleného jednou svíčkou. » (Andreï Makine, Francouzský testament, traduit par Vlasta Dufková) ${ }^{25}$

« D'autant que celui-ci quitta son fauteuil de cuir, ferma solennellement la porte du bureau en faisant doucement tourner la clé dans la serrure à la manière de celui qui prépare ses effets, tira les épais rideaux en daim et ne laissa allumée qu'une lampe de chevet sur un accoudoir.» (Pierre Assouline, Double vie $)^{26}$

23 Néanmoins, ne...que n’entraîne pas toujours la valeur numérale de un, comme c'est le cas dans cet exemple-ci : « Je fis péniblement le tour du château, plongeant parfois jusqu'aux chevilles dans l'eau d'une flaque, et, passé une sorte de marécage qui n'eût été en temps normal qu’une couche de terreau fraîchement retourné, [...] (André Pieyre de Mandiargues, Soleil des Loups) traduit comme «S velkými obtížemi jsem obešla celý zámek, chvílemi zapadajíc až po kotníky do kaluží, a když jsem se vyhrabala z jakéhosi bahniska, což za normálních okolností zřejmě byl jen [Ø] čerstvě navezený kompost, [...] » (André Pieyre de Mandiargues, Vlčí slunce, traduit par Ladislav Šerý).

24 Ces expressions servent à renforcer la valeur numérale de un, qui a perdu au cours de l'évolution du français sa force expressive originelle (à l'origine, son prédécesseur unus était numéral) (Carlier 2001 : 72, 77-78).

25 À la différence de la traductrice, nous ne percevons pas dans la phrase de départ la valeur numérale de un : nous désignerions un comme article et non comme numéral.

26 Nous avons désigné cette occurrence de un comme un " cas douteux » de un numéral (pour l'explication de cette appellation, voir le raisonnement ci-après), cela surtout grâce à la présence de ne...que : pourtant, léventuelle valeur numérale de un dans ce cas n’est aucunement reflétée dans la traduction tchèque. 
«Tím spíš, když se Jean zvedl z koženého křesla, slavnostně zavřel dveře pracovny a potichounku otočil klíčem jako někdo, kdo chystá překvapení, zatáhl těžké sametové závěsy a nechal svítit jen [Ø] malou lampičku u křesla. » (Pierre Assouline, Dvojí život, traduit par Šárka Belisová)

C'est pourquoi, après avoir essayé de relever les occurrences de un numéral dans l'Échantillon 1, nous les avons réparties en cas sûrs, où il s'agissait apparemment de un numéral, et en cas douteux, où un pouvait être interprété soit comme article, soit comme numéral. Maintenant, consacrons un instant à l'analyse de nos résultats le bilan desquels nous pouvons formuler comme suit :

- 466 occurrences du quantifiant un

- dont 64 occurrences de un à valeur numérale (14\% sur 466 occurrences)

- dont 35 cas douteux (8\% sur 466 occurrences)

- dont 29 cas sûrs = un numéral (6\% sur 466 occurrences)

Pour illustrer les différents cas de figure, c'est-à-dire d'un côté les cas sûrs, dont nous allons nous occuper davantage, et de l'autre côté les cas douteux, que nous n'allons plus aborder, nous proposons un exemple pour chacun :

« Mais surtout, il posa devant nous une bouteille de vin.» (Andreï Makine, Le Testament français)

«Ale hlavně před nás postavil [Ø] láhev vína. » (Andreï Makine, Francouzský testament, traduit par Vlasta Dufková)

Dans cette phrase, l'interprétation numérale de une est possible : nous désignons donc ce cas comme douteux.

« Là, suspendu d'une main entre le wagon des bagages et le tender, de l'autre il décrocha les chaînes de sûreté [...]» (Jules Verne, Le tour du monde en quatre-vingt jours)

«Pak zůstal viset na jedné ruce mezi zavazadlovým vozem a tendrem a druhou rukou začal odpojovat zajištovací řetězy [...] » (Jules Verne, Cesta kolem světa za osmdesát dní, traduit par Josef Pospíšil)

$\mathrm{Vu}$ le fait que, d'habitude, l'homme n'a que deux mains et que l'interprétation « être suspendu d'une quelconque main » paraîtrait sans doute improbable, il s'agit sûrement de un numéral (donc d'un cas sûr).

En nous focalisant sur les cas sûrs, il est frappant que dans 19 cas (sur 29) un numéral accompagne un nom exprimant une "mesure », p. ex. mètre (1 fois), litre (1 fois), mais aussi goutte (2 fois), fois ( 2 fois) ou douzaine (1 fois). Le plus souvent, nous avons trouvé des " unités » de mesure du temps (12 occurrences au total) : an (4 fois), heure ${ }^{27}$ ( 3 fois), minute ( 2 fois), seconde (2 fois), demi-siècle (1 fois). Il est à remarquer que dans les traductions tchèques, à l'exception 
de $2 \operatorname{cas}^{28}$ (sur 12), un numéral accompagnant une indication chronologique n’est pas exprimé « directement » au niveau lexical ${ }^{29}$, par exemple :

«Moi je n'ai aucune situation, toi tu es au chômage, comment va-t-on vivre dans un an ?» (Bernard Werber, Les Fourmis)

« Já nemám žádné postavení, ty jsi přišel o práci, jak budeme žít za [Ø] rok? » (Bernard Werber, Mravenci, traduit par Richard Podaný)

Lorsque un numéral (nous ne parlons toujours que des cas sûrs) est exprimé au niveau lexical en tchèque (ce qui a lieu dans 12 occurrences), c'est par l'intermédiaire des expressions suivantes : jeden ( 8 fois), jediný ( 3 fois) et celý ( 1 fois). Sur ces 12 occurrences, 10 fois la valeur numérale de un est accentuée au moyen d'une expression comme ne...que, pas (un), au moins, sans, seul ou presque. Voici un exemple :

«La deuxième ligne n'a même pas le temps de se mettre en place, les artilleuses sont toutes saisies à la gorge et décapitées sans avoir pu lâcher une goutte d'acide. » (Bernard Werber, Les Fourmis) «Druhá ani neměla čas je vystřídat a už byly všechny dělostřelkyně chyceny pod krky a otrokářky jim ustř́haly hlavy, ještě než mohly vypálit jedinou kapku kyseliny. » (Bernard Werber, Mravenci, traduit par Richard Podaný)

De plus, en confrontant nos résultats avec la théorie de Wilmet, à part 2 exceptions, où l'extensité de un semble augmenter grâce au déclencheur cotextuel, toutes les occurrences de un numéral (y inclus les cas douteux) coïncident justement avec les occurrences de un relevant de l'extensivité partitive, précisément de l'extensité égale à 1 .

Eu égard à la fréquente impossibilité de distinction entre $u n$ article et $u n$ numéral $^{30}$, que nous venons de signaler, ne faudrait-il pas reconsidérer la question de la polysémie/homonymie de ce petit mot ? Peut-être, est-il " plus polysémique » qu'il ne le semble au premier abord : sa valeur numérale toujours présente en latence ou sous narcose, comme le dirait Umberto Eco, pourrait justifier le bien-fondé de cette hypothèse.

28 En voici un : «Oublier l'atroce misère du monde, Yanek, se laisser aller enfin, une heure, une seule petite heure d'égoïsme, imagines-tu cela ? (Albert Camus, Carnets II) traduit comme « Zapomenout na krutou bídu světa, Janku, konečně pustit všechno z hlavy, jen jednu jedinou hodinku být sobecký, dovedeš si to představit? » (Albert Camus, Zápisníky II, traduit par Vlasta Dufková et Josef Mlejnek). Peut-être, dans ce cas, l'expression lexicale « directe » de un numéral est-elle due à la présence de l'adjectif seule.

29 Ceci est en accord avec l’affirmation de Josef Zubatý dans son article « Číslovka »jeden«» : « Jména, vyjadřující určitou míru nebo určité množství čehokoli, kladou se nejčastěji sama, bez číslovky jeden, má-li se rozuměti jediná jednotka oné míry, onoho množství [...] [Les noms exprimant une mesure déterminée ou une quantité déterminée de quoi que ce soit se trouvent le plus souvent tous seuls, sans l'adjectif numéral jeden, lorsqu’on sous-entend une seule unité de cette mesure, de cette quantité] » (Zubatý 1918).

30 Surtout dans une phrase affirmative où la forme négative ( aucun » pour un article vs. " pas un » pour un numéral) ne facilite pas la distinction entre les deux. 


\section{En guise de conclusion}

Concentrée sur l'étude des moyens d'expression en tchèque de ce déterminant français, qui sont plus nombreux et plus raffinés qu'il ne semble au premier abord (on exploite les niveaux lexical, morphologique et syntaxique, souvent en les combinant), nous avons dû nous rendre à l'évidence : grâce aux traductions du français vers le tchèque composant notre Échantillon 1, on mesure à quel point la distinction entre un article indéfini et un adjectif numéral cardinal est souvent difficile à opérer. Par contre, ce que ces un ont en commun, c'est leur valeur originale et - disons-le clairement - la plus fréquente ${ }^{31}$ : l'expression d'un résidu d'extension (Wilmet 2007 : 148), le résidu prenant la forme d'une unité (quoique abstraite).

De plus, il est à remarquer que, dans la théorie de Wilmet, les trois valeurs de l'extensité de un (cf. Fig. 1 : Extensité vs. extensivité) maintiennent cette valeur numérale d’origine héritée du latin unus : l'extensivité partitive où l'extensité ne dépasse pas 1 la conserve de par sa nature même, l'extensivité partitive augmentée le fait par l'intermédiaire de l'addition caractéristique pour son mécanisme $1+1+1 \ldots+1=n<t$, l'extensivité extensive le fait par contre au moyen de la multiplication, propre à son mécanisme $1 \times n \rightarrow t$. De plus, tout cela vaut tant pour un dit article indéfini, que pour un dit adjectif numéral. Si on pouvait parler en termes de sémantique et comme nous avons pu nous en apercevoir dans les traductions, le sème inhérent à ce petit mot semble être unique, quelque soit la «valeur activée » du déterminant un. Bref, et si un nétait finalement qu'un seul... ?

\section{Références bibliographiques}

Carlier, A. (2001). La genèse de l'article un. Langue française, 130, 65-88. In: http://www.persee.fr/web/ revues/home/prescript/article/lfr_0023-8368_2001_num_130_1_1027 [2014-03-31]).

Fournier, J.-M. (2005). Classe et Individu dans les grammaires générales tardives. In J. Bourquin (coord.), Les prolongements de la Grammaire Générale en France au XIXe siècle : colloque de Besançon, 19-21 septembre 2002 (pp. 105-118). Besançon: Presses Universitaires de Franche-Comté.

Franckel, J.-J. (1998). Référence, référenciation et valeurs référentielles. Sémiotiques, 15, 61-84. In : http:// www.revue-texto.net/Parutions/Semiotiques/SEM_n15_4.pdf [2014-04-05].

Hirschová, M. (2006). Pragmatika v češtině. Olomouc: Univerzita Palackého v Olomouci.

Koláčková, K. (2014). Le mot UN en questions. Mémoire de maitrise sous la direction de Christophe Cusimano. Brno: Masarykova univerzita.

« Konkordance ». In: http://wiki.korpus.cz/doku.php/pojmy:konkordance [2014-04-03].

« Korpus InterCorp ». In: http://ucnk.ff.cuni.cz/intercorp/?req=page:info [2014-04-02].

Mathesius, V. (1975). A Functional Analysis of Present Day English on a General Linguistic Basis. J. Vachek (Ed.), The Hague: Mouton.

Nádvorníková, O., \& Vavř́n, M. (2013). Korpus intercorp_fr, verze 6 z 8. 4. 2013. Praha: Ústav Českého národního korpusu FF UK. In: http://www.korpus.cz/intercorp [2014-03-14].

« Paralelní korpus InterCorp ». In: http://wiki.korpus.cz/doku.php/pojmy:paralelni [2014-04-03].

31 L'écrasante majorité d’occurrences de un de notre Échantillon 1 correspondaient justement à extensité égale à 1. 
Radina, O. (1977). Francouzština a čeština: systémové srovnání dvou jazykư. Praha: Státní pedagogické nakladatelství.

Rey-Debove, J., \& Rey, A. (2012). Le nouveau Petit Robert: dictionnaire alphabétique et analogique de la langue française [version électronique].

Rosen, A., \& Vavř́n, M. (2013). Korpus intercorp_cs, verze 6 z 8. 4. 2013. Praha: Ústav Českého národního korpusu FF UK. In: http://www.korpus.cz/intercorp [2014-03-24]

Wilmet, M. (2007). Grammaire critique du français. $4^{\mathrm{e}}$ éd. Bruxelles: De Boeck \& Larcier.

Zubatý, J. (1918). Číslovka »jeden«. Naše řeč. 2, 4. In: http://nase-rec.ujc.cas.cz/archiv.php?art=237 [201404-18]. 